\title{
CONFORMIDADE DO POSTO DE OPERAÇÃO DE PULVERIZADORES AUTOPROPELIDOS
}

\author{
André Luis Casali ${ }^{1}$, Marcelo Silveira de Farias², José Fernando Schlosser ${ }^{3}$, Leonardo Nabaes Romano ${ }^{4}$,
} Daniel Uhry ${ }^{5}$

\section{RESUMO}

As operações com pulverizadores autopropelidos exigem um grande esforço físico e mental dos operadores. A correta disposição dos comandos aumenta, consideravelmente, a eficiência da operação. Em vista disso, este trabalho teve como objetivo identificar e comparar a localização dos comandos de uso frequentes e raros pelo operador em cinco pulverizadores autopropelidos, segundo a norma ISO 15077. Foram medidas as coordenadas dos principais comandos de operação em relação ao SIP (Seat Index Point). A análise revelou que o número de comandos decresce à medida que o nível tecnológico do pulverizador aumenta. Entre os comandos de acionamento frequentes, presentes em todos os pulverizadores, destacam-se os modelos 4730 e Patriot 350, que obtiveram 100\% dos comandos dentro da Zona de Conforto nos dois perfis analisados. Os pulverizadores Impactus 2750, Patriot 350 e Gladiador 3000 não obtiveram comandos localizados na zona inacessível em nenhum dos dois perfis analisados. Desta forma, podemos concluir que os modelos avaliados apresentam grande variação no número dos comandos, que se encontram, na maioria dos casos, dentro da zona de conforto para pulverizadores com maior nível tecnológico e em menor expressão em pulverizadores com menor nível tecnológico.

Palavras-chave: ergonomia, conforto, operador

\section{ABSTRACT \\ CONFORMANCE OF OPERATING POST OF SELF-PROPELLED SPRAYERS}

The operation of self-propelled sprayers requires considerable physical and mental efforts of operators. The correct layout of controls significantly increases operating efficiency. This study aimed to verify and compare the location of more as well as less frequently used operating commands in five brands of self-propelled sprayers, according to the ISO 15077 standards. The coordinates of the main operating commands were measured in relation to the Seat Index Point. The data revealed that the number of commands decreases with increasing technological level of the equipment. The models 4730 and Patriot 350 had 100\% of frequent access commands controls within the Comfort Zone in both profiles, while the models Impactus 2750, Patriot 350 and Gladiator 3000, had commands located in Inaccessible Zone. Thus we can conclude that there is great variation in the models as to the number of commands, are located within the Comfort Zone in sprayer models with higher technological level and to a lesser extent in models of low technological level.

Keywords: ergonomic, comfort, operator

\footnotetext{
Recebido para publicação em 09/01/2011. Aprovado em 16/09/2011.

1- Eng. Agrônomo Mestrando PPGEA UFSM, Santa Maria - RS, e-mail: andrcasali@yahoo.com.br

2- Academico de Agronomia UFSM, Santa Maria - RS, e-mail: silveira_farias@hotmail.com

3- Prof. Doutor Departamento Engenharia Rural, Santa Maria - RS, e-mail: josefernandoschlosser@gmail.com

4- Prof. Doutor Departamento Engenharia Rural, Santa Maria - RS, e-mail: leo.nabaes.romano@gmail.com

5- Eng. Agônomo Doutorando PPGEA UFSM, Santa Maria - RS, e-mail: d_uhry@hotmail.com
}

\section{REVENG}




\section{INTRODUÇÃO}

No Brasil, o consumo de agrotóxicos em 2009 apontou um volume de 1,06 milhões de toneladas, segundo dados do Sindicato Nacional da Indústria de Produtos para a Defesa Agrícola (SINDAG, 2010). A utilização destes produtos tem por objetivo assegurar o potencial produtivo das plantas cultivadas na agricultura, reduzindo o efeito nocivo de agentes causadores de danos às culturas.

Os pulverizadores hidráulicos são os principais equipamentos utilizados para pulverização. Atualmente, existem diversos modelos que vão desde os mais simples, do tipo costal, bastante utilizado em pequenas propriedades, até os autopropelidos, que possuem alta tecnologia embarcada e grande capacidade operacional, com elevada qualidade na pulverização.

A operação dos pulverizadores autopropelidos assim como qualquer outra máquina agrícola é uma tarefa árdua, exigindo esforço físico e mental por parte do operador, resultando em fadiga no decorrer de uma jornada de trabalho. A intensidade desta fadiga irá depender da qualidade do posto de operação da máquina. Níveis elevados de fadiga aumentam, consideravelmente, o risco de acidentes e as perdas econômicas na cadeia produtiva.

O posto de operação é o local de interação entre homem e máquina, composto por órgãos de comando e elementos que auxiliam na condução da máquina no ambiente de trabalho. Segundo a norma ISO 15077 (1996), comando é um dispositivo que ao ser acionado promove uma resposta na máquina ou implemento que esteja acoplado. Berasategui (1997) afirma que um posto de operação bem projetado deve ser composto por um local de trabalho organizado de acordo com a segurança e a natureza do operador. Os comandos existentes no posto de operação de um pulverizador autopropelido são variados, como, por exemplo, botões, alavancas, volante e pedais. Algumas máquinas apresentam dispositivos de comandos com multifunções (alavanca de multifunções ou Joystick), geralmente, posicionados à direita no posto de operação.

As dimensões do posto de operação de uma máquina agrícola devem respeitar o fator humano, seguindo os padrões estabelecidos pelas normas ISO (International Organization for Standardization), ABNT (Associação Brasileira de Normas Técnicas) e ASAE (American Society of Agricultural Engineers). Essas normas definem parâmetros a serem utilizados em projetos de máquinas agrícolas, definindo as dimensões recomendadas, considerando a presença do operador no assento, estabelecendo distâncias máximas e mínimas de acesso aos comandos e delimitando áreas onde os comandos de operação devem ser posicionados para permitir conforto e segurança ao operador.

Para simular o operador sentado no posto de operação de uma máquina agrícola, adota-se um ponto de referência sobre o assento do operador, conhecido como Seat Index Point (SIP), traduzido como o Ponto de Indexação do Assento, definido pela norma ISO 5353 (1999). Já a norma ISO 15077 (1996) delimita áreas de alcance para o operador sentado em relação ao mesmo ponto de referência, abrangendo todos os comandos presentes no posto de operação de máquinas agrícolas autopropelidas.

Devido ao grande número de pulverizadores autopropelidos comercializados no Brasil e à falta de trabalhos que avaliem a disposição dos comandos no posto de operação dessas máquinas, o objetivo deste trabalho foi avaliar e comparar a conformidade dos postos de operação de cinco modelos de pulverizadores autopropelidos comercializados no Brasil com a norma ISO 15077, que delimita a área de alcance dos comandos, considerando sua frequência de uso.

\section{MATERIAL E MÉTODOS}

Entre os cinco pulverizadores utilizados para a realização deste trabalho, foram avaliados os modelos que apresentavam maior reservatório de calda. E entre esses modelos, foram analisados os pulverizadores Case IH Patriot 350 (reservatório de calda com capacidade de 3500 litros), John Deere 4730 (reservatório de calda com 3028 litros), Jacto Uniport3000(reservatório decalda com3000litros), Stara Gladiador 3000 (reservatório de calda com 3000 litros) e Jan S/A Impactus 2750 (reservatório de calda com 2750 litros). Foram utilizados pulverizadores novos ou com poucas horas de uso, 
desta forma, todos os comandos localizados na máquina não apresentaram adaptações feitas pelos proprietários. Os pulverizadores autopropelidos foram selecionadas a partir de visitas a algumas fábricas, concessionárias e produtores proprietários de pulverizadores que atendiam aos requisitos, anteriormente, preestabelecidos.

Os instrumentos utilizados para a obtenção dos dados no posto de operação foram prumo, fita métrica, nível de bolha, pesos metálicos, máquina fotográfica, esquadro e o Software autoCAD utilizado para a representação gráfica dos comandos. Adotando os critérios descritos na norma ISO 5353, também foi utilizado o SIP (Figura 1), dispositivo já descrito acima, para a determinação do ponto de referência do assento.

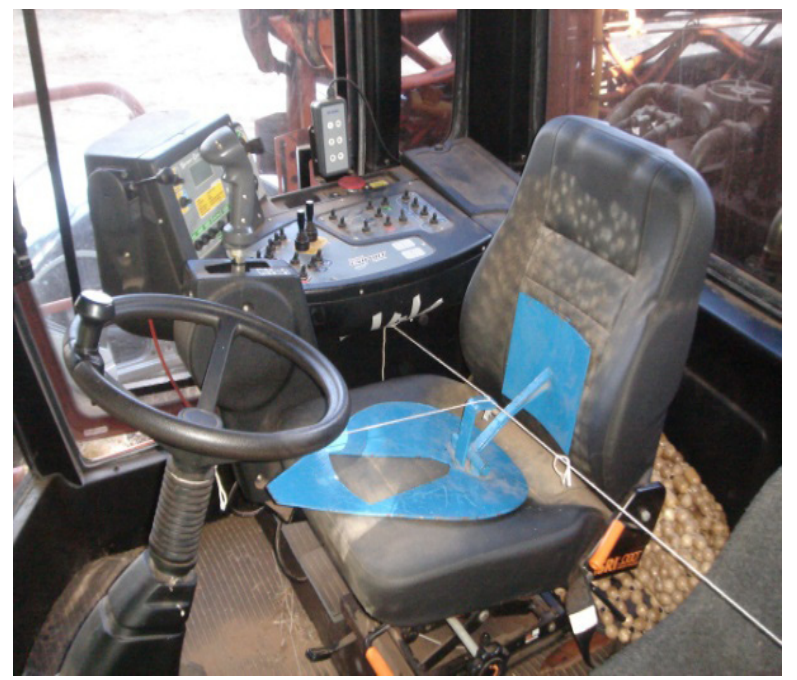

Figura 1. Linhas de referência para a determinação do SIP no assento do operador.

A metodologia adotada para aquisição dos dados foi proposta por Rozin (2004), com adaptações às condições do trabalho. As medidas ergonométricas no posto de operação, em relação ao SIP, foram tomadas com o volante e o assento em uma posição intermediária nos planos vertical e horizontal. Após seu posicionamento, o SIP era posicionado sobre o assento, sendo, posteriormente, adicionadas massas metálicas padronizadas até atingir a massa desejada (contabilizando-se o dispositivo), simulando o peso de um operador de $75 \mathrm{~kg}$. Logo após, foram medidas as distâncias dos comandos até o SIP em três eixos (X, Y e Z).

Foram mensuradas as posições de todos os comandos de operação dos pulverizadores avaliados, fossem eles de acionamento frequente ou raro. Segundo a Norma ISO 15077, os comandos de operação são classificados segundo sua frequência de utilização em frequentemente e raramente acionados. O comando é classificado como de acionamento frequente quando utilizado em um intervalo igual ou inferior a cinco minutos durante a operação da máquina. Nos casos cujo intervalo de acionamento é maior que cinco minutos, esses comandos passam a ser classificados como de acionamento raro. Os comandos de acionamento frequente devem estar localizados na zona de conforto do posto de operação, já os raramente utilizados podem se situar tanto na zona de conforto como na zona de acesso, porém, quando estiverem localizados na zona de acesso, eles não devem demandar força de acionamento maior que a necessária para acionar os comandos localizados na zona de conforto. Quando os comandos não estão nas zonas citadas, eles são classificados como inacessíveis. Na Figura 2 mostram-se as áreas de alcance no perfil vertical longitudinal (PVL) e no perfil superior horizontal (PSH).

Com a utilização desses modelos como parâmetros, foram identificados, nas figuras confeccionadas no software autoCAD, quais comandos estavam nas zonas de conforto, acessíveis ou inacessíveis, tanto no perfil longitudinal vertical (Figura 3A) quanto no PSH (Figura 3B).

Os resultados obtidos com as avaliações ergonômicas nos postos de operação foram comparados entre si com o objetivo de produzir mais informações que pudessem contribuir para a elaboração de trabalhos subsequentes, mostrando a grande importância dos parâmetros de ergonomia e segurança na elaboração e execução de futuros projetos na área de máquinas agrícolas, possibilitando um constante aperfeiçoamento tecnológico dos pulverizadores autopropelidos fabricados no país.

\section{RESULTADOS E DISCUSSÃO}

Houve grande variação no número de comandos existente nos postos de operação dos pulverizadores avaliados, variando de 26 a 49 comandos. Esta variação se deve ao nível tecnológico empregado na máquina, em que os pulverizadores com maior nível tecnológico apresentaram menos comandos. 
Entre os comandos presentes na maioria dos pulverizadores avaliados, definidos como sendo de acionamento frequente, podemos destacar o volante, o controlador de velocidade, o Joystick, o acionamento do GPS, o acionamento da bomba de pulverização, o ajuste da altura total das barras e o ajuste da altura da barra esquerda e direita. $\mathrm{O}$ volante foi o comando que mais esteve presente na zona de conforto dos cinco pulverizadores amostrados, e todos apresentaram este comando dentro desta área para o PSH; já para o perfil longitudinal vertical, apenas o pulverizador Gladiador 3000 não apresentou este comando dentro da zona de conforto. O Joystick é um importante instrumento de controle de diversos comandos presentes nas máquinas, pois agrupa

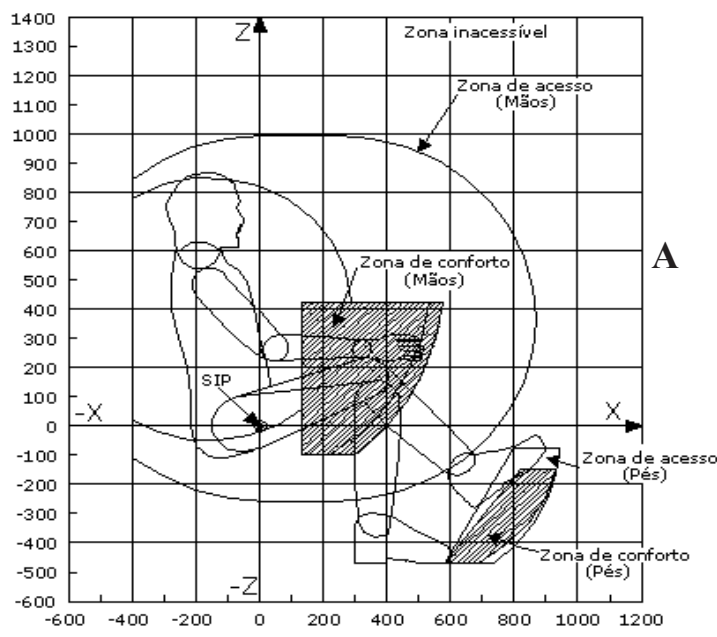

uma série de funções em um único local. No caso de alguns pulverizadores agrícolas, as principais funções encontradas no Joystick são a regulagem da altura das barras, o acionamento do sistema de pulverização e o acionamento do GPS. Nas máquinas avaliadas, o Joystick esteve presente em quatro dos cinco modelos avaliados e, em todos os modelos em que foi encontrado, ele estava posicionado dentro da zona de conforto nos dois perfis. No modelo em que não foi encontrado Joystick, os botões responsáveis pelo controle da altura total das barras e altura das barras direita e esquerda estavam presentes na zona de acesso no PSH; já no PVL, os três comandos responsáveis pela altura das barras estavam dentro da zona de conforto.

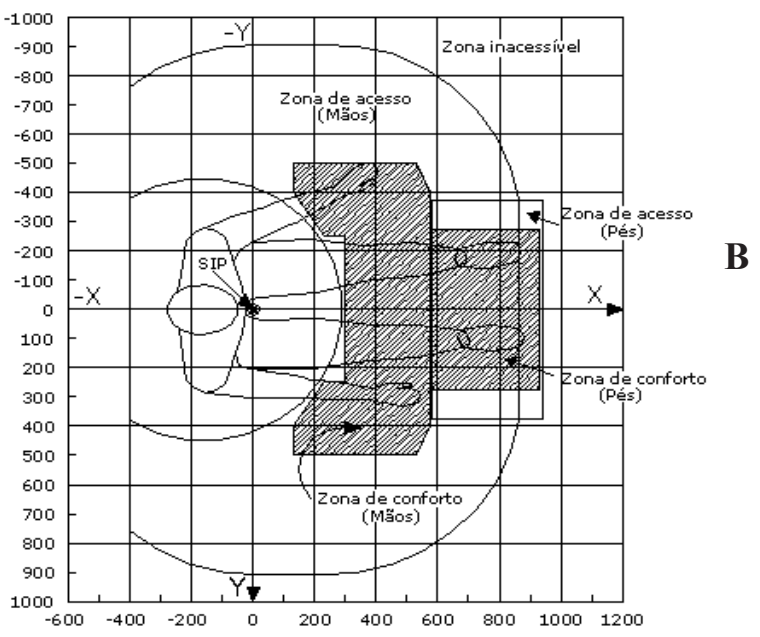

Fonte: ISO 15077

Figura 2. Regiões de alcance do operador sentado no plano longitudinal vertical (A) e superior horizontal (B).
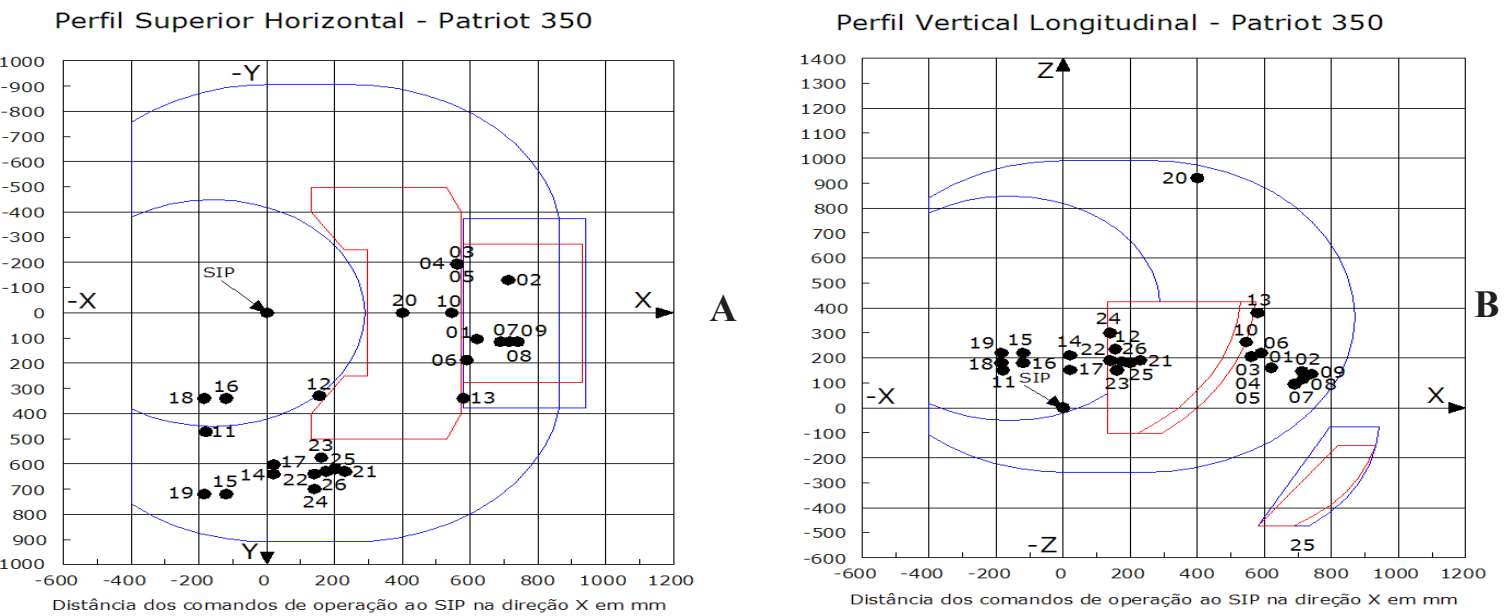

Figura 3. Localização dos comandos de operação do pulverizador autopropelido da Case IH Patriot 350 no PVL (A) e PSH (B). 
Em relação ao controlador de velocidade, os pulverizadores Case Patriot 350, John Deere 4730 e Jan Impactus 2750 têm o controlador de velocidade dentro da zona de conforto nos dois perfis; já o pulverizador Stara Gladiador 3000 tem esse comando dentro da zona de conforto apenas para o PVL. No PSH, este comando está na zona de acesso, por outro lado, no pulverizador Jacto Uniport 3000, o controlador de velocidade localizase dentro da zona de conforto no PSH e na zona de acesso no PVL.

A interface de acesso ao Sistema de Posicionamento Global (GPS) é uma das ferramentas mais importantes utilizadas no processo de pulverização agrícola. Com o auxílio deste sistema, o operador conduz a máquina de maneira mais eficiente, reduzindo as sobreposições e, consequentemente, melhorando a qualidade da aplicação. Nesse sentido, o acesso à interface do GPS localizou-se na zona de conforto tanto no PSH quanto no vertical longitudinal nos pulverizadores JD 4730, Patriot 350 e Impactus 2750. Nos pulverizadores Gladiador 3000 e Uniport 3000, os comandos localizavam-se na zona de acesso nos dois perfis, anteriormente, citados. Outro comando de fundamental importância é o acionamento do sistema de pulverização, responsável por iniciar o fracionamento do líquido pelas pontas. Dos pulverizadores avaliados, destacam-se os modelos Patriot 350, JD 4730 e Uniport 3000, que apresentaram o comando dentro da zona de conforto nos dois perfis analisados; já o pulverizador Impactus 2750 apresentou esse comando dentro da zona de conforto apenas no PVL, estando na zona de acesso para o PSH. O pulverizador Gladiador 3000 apresentou o comando na zona de acesso para os dois perfis avaliados.

O pulverizador Case IH Patriot 350 se destacou por apresentar o menor número de comandos de operação avaliados, uma característica é positiva, pois quanto menos comandos menor a chance de perda de atenção por parte do operador. Dos 26 comandos encontrados, em nenhum dos dois perfis eles foram localizados na zona inacessível. Desse total, 12 classificaram-se como comandos de acionamento frequente e 14 como de acionamento raro. Com base na análise do $\mathrm{PSH}, 25 \%$ dos comandos considerados de uso frequente situavam- se na zona de conforto e $75 \%$ na zona de acesso. Já dos comandos classificados como de acionamento raro, apenas $28,57 \%$ figuravam na zona de acesso, e os restantes $71,43 \%$, na zona de conforto, porém não haveria necessidade de esses altos percentuais de comandos estarem localizados nessa zona, pois eles não são acessados com frequência. Nesse caso, ocorreu uma inversão, pois os a maioria dos comandos que deveriam estar localizados na zona de acesso (comandos de acionamento raro) estavam na zona de conforto, e a maioria dos que deveriam estar na zona de conforto (comandos de acionamento frequente) estavam na zona de acesso. A análise dos comandos de acesso frequente e raro está em maior conformidade com a norma no PVL. Nesse perfil, 66,67\% dos comandos de frequente acionamento localizavam-se na zona de conforto e $33,33 \%$ na zona de acesso. Para os comandos de acionamento raro, $92,86 \%$ estavam presentes na zona de acesso (Figura 4).

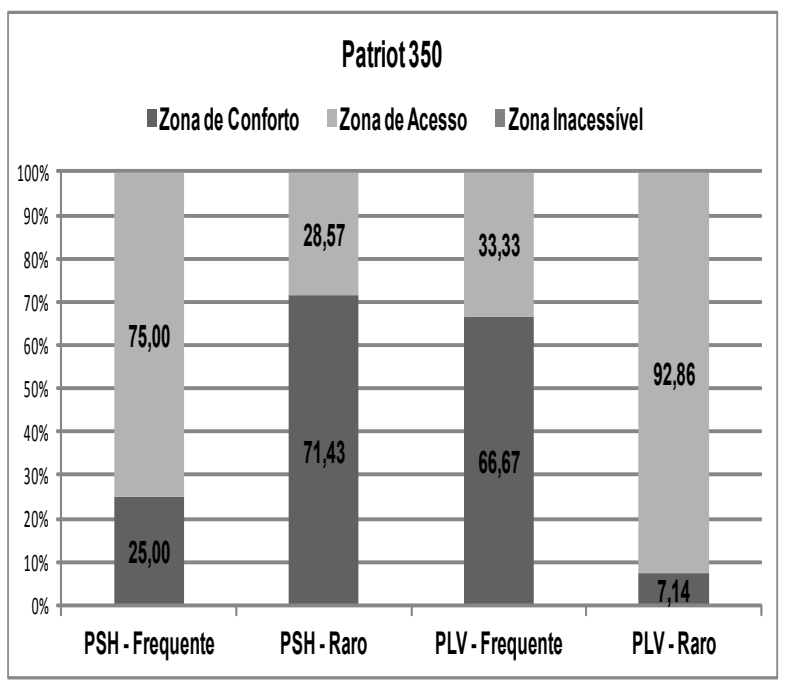

Figura 4. Distribuição de comandos de acionamento frequente e raro nos perfis superior horizontal e vertical longitudinal do pulverizador Case Patriot 350.

O modelo Gladiador 3000 produzido pela empresa Stara disponibiliza 33 comandos para o operador, sendo 19 de acesso frequente e 14 de acesso raro. Quando avaliado o PSH, apenas $21,05 \%$ dos comandos de operação considerados de acionamento frequente estavam localizados na zona de conforto e $78,95 \%$, na zona de acesso. 
Quando avaliados os comandos de uso raro, $85,71 \%$ deles estavam posicionados na zona de conforto e apenas $14,29 \%$, na zona de acesso. Na avaliação do PVL, os comandos de acionamento frequente estavam localizados $52,63 \%$ na zona de conforto e $47,37 \%$ na zona de acesso. Já dos comandos de acionamento raro, $92,86 \%$ estavam localizados na zona de acesso. Não existem comandos localizados na zona inacessível (Figura 5).

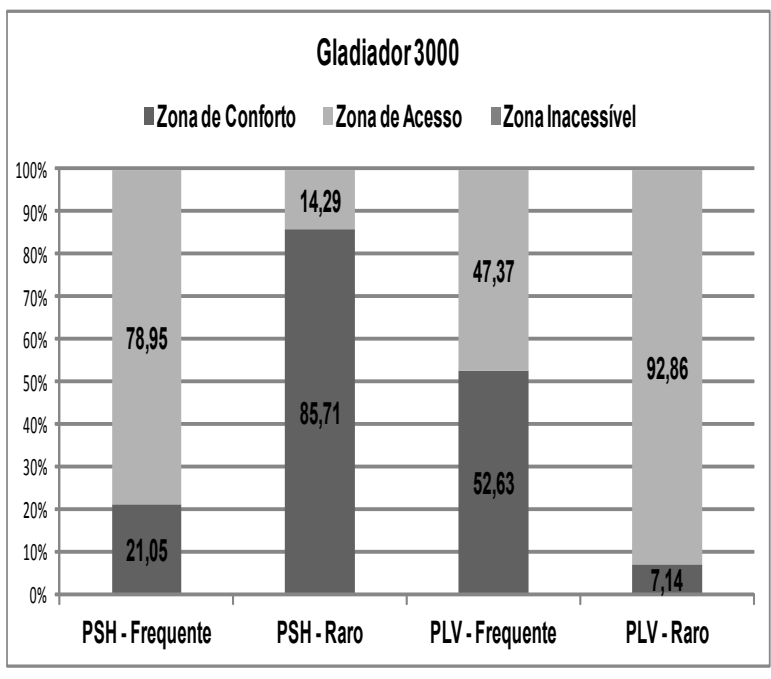

Figura 5. Distribuição dos comandos de acionamento frequente e raro nos perfis superior horizontal e vertical longitudinal do pulverizador Stara Gladiador 3000.

O modelo Impactus 2750, da fabricante Jan S/A, apresentou 42 comandos de operação, divididos em 26 comandos de acesso frequentes e 16 de acesso raro. Quando avaliado o PSH, 61,64\% dos comandos de operação de acesso frequente localizavam-se na zona de conforto; já para os de acesso raro, apenas $18,75 \%$ estavam disponíveis na zona de acesso. Analisando o PVL, $80,77 \%$ dos comandos de acesso frequente estavam em conformidade com a norma ISO 15077, isto é, localizados na zona de conforto; já para os comandos de acesso raro, $31,25 \%$ estavam disponíveis na zona de acesso. Este pulverizador nos chama a atenção por apresentar a maioria de seus comandos na zona de conforto, independentemente de serem de acesso frequente ou raro (Figura 6). Essa característica é fundamental para que o operador mantenha a concentração durante pulverização, demandando menos esforço para acionar os comandos durante a jornada de trabalho.

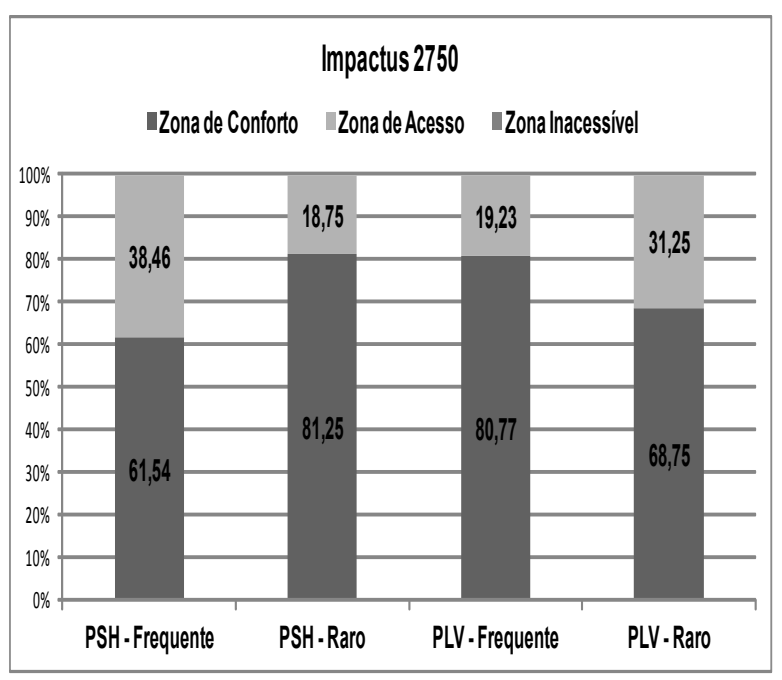

Figura 6. Distribuição dos comandos de acionamento frequente e raro nos perfis superior horizontal e vertical longitudinal do pulverizador Jan Impactus 2750.

O modelo 4730, fabricado pela John Deere, disponibiliza um total de 30 comandos de operação, sendo 14 de acesso frequente e 16 raramente acessados. Analisando o PSH, 42,86\% dos comandos de acesso frequente estavam localizados na zona de conforto, e os outros $57,14 \%$, na zona de acesso. Mais de $60 \%$ dos comandos de acesso raro estavam alocados na zona de acesso. Quando analisamos o PVL, constatamos que existem poucos comandos de operação distribuídos na zona inacessível, $7,14 \%$ e $6,25 \%$ para comandos de acesso frequente e raro, respectivamente. Nesse caso, um dos comandos encontrados na zona inacessível era o pedal de freio, que é um importante comando de operação. É importante destacar que esse modelo de pulverizador oferece ao operador um grande número de posições para ajuste, tanto do acento quanto do volante, o que pode mudar a disposição dos comandos em relação ao SIP, consequentemente, enquadrando esses comandos na zona de acesso ou de conforto. No entanto, como as avaliações foram feitas utilizando posições médias do acento e do volante, esses comandos se apresentaram na zona inacessível. Para os de comandos de acesso frequente, $71,43 \%$ estão dispostos na zona de conforto e para os de acesso raro, 62,50\%, na zona de acesso (Figura 7). 


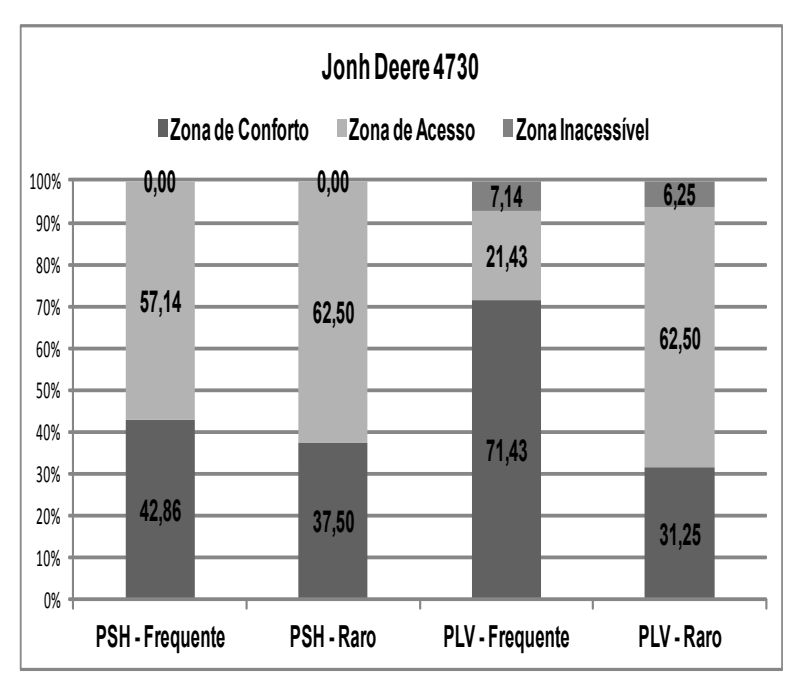

Figura 7. Distribuição dos comandos de acionamento frequente e raro nos perfis superior horizontal e vertical longitudinal do pulverizador John Deere 4730.

O pulverizador Uniport 3000 da marca Jacto apresentou o maior número de comandos de operação, 49 no total, classificados em 29 comandos de acesso frequente e 20 de acesso raro. Analisando o $\mathrm{PSH}, 68,97 \%$ dos comandos de acesso frequente estavam localizados na zona de conforto e os $31,03 \%$ restantes na zona de acesso. Já para os comandos de acesso raro, este pulverizador se mostrou $85 \%$ em conformidade com a norma, estando esses comandos alocados na zona de acesso. Quando analisado o PVL, mais de $80 \%$ dos comandos de acesso frequente estavam localizados na zona de conforto, entre eles, podemos destacar o Joystick, acionamento do sistema de orientação por GPS e o volante, comandos fundamentais para uma eficiente pulverização. Quanto aos de acesso raro, 60\% estavam localizados na zona de acesso, como, por exemplo, o acelerador manual, que se enquadra nessa classificação, pois no processo de pulverização a rotação do motor é selecionada e sua mudança é normalmente efetuada após cinco minutos. Os restantes $25 \%$ estavam na zona de conforto e apenas $15 \%$ na zona inacessível, em que se destacaram o controle do ar condicionado e a tela de visualização do sistema GPS. Na Figura 8 mostra-se a distribuição dos comandos de operação do pulverizador Uniport 3000 nos perfis avaliados.

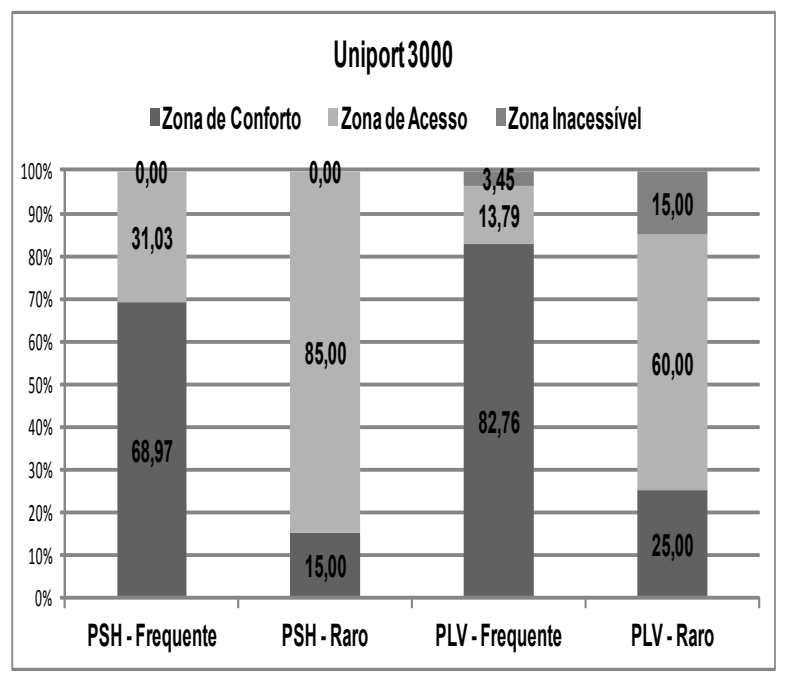

Figura 8. Distribuição dos comandos de acionamento frequente e raro nos perfis superior horizontal e vertical longitudinal do pulverizador Jacto Uniport 3000.

Fazendo uma análise comparativa entre os cinco pulverizadores autopropelidos avaliados nesse trabalho, conclui-se que, pela análise do $\mathrm{PSH}$, os comandos de operação frequente estavam presentes em maior número na zona de conforto no pulverizador Uniport 3000, estando $68,97 \%$ dos comandos localizados nesta zona. Já na zona de acesso dos comandos, os pulverizadores Gladiador 3000 e o Patriot 350 foram os que obtiveram os maiores valores percentuais, $78,95 \%$ e $75,0 \%$, respectivamente. Já com relação aos comandos de acesso raro, os pulverizadores Gladiador 3000, Impactus 2750 e Patriot 350 se destacaram, estando acima de $70 \%$ dos comandos na zona de conforto. Porém, devido a estes comandos não serem acessados de forma tão frequente, não haveria necessidade de se localizarem nesta zona. Logo, mais uma vez, o pulverizador Uniport 3000 da Jacto é o que melhor atende à norma ISO 15077 , visto que $85 \%$ dos comandos de acesso raro estão na zona de acesso. Novamente, é importante salientar que nenhum pulverizador possui comandos de acesso frequente na zona inacessível (Figura 9). Dessa forma, verificamos que os pulverizadores avaliados propiciam um adequado posicionamento dos comandos para esse perfil, indicando que existe elevada qualidade na disposição dos comandos, isso indiretamente proporciona maior ergonomia durante as operações de pulverização. 


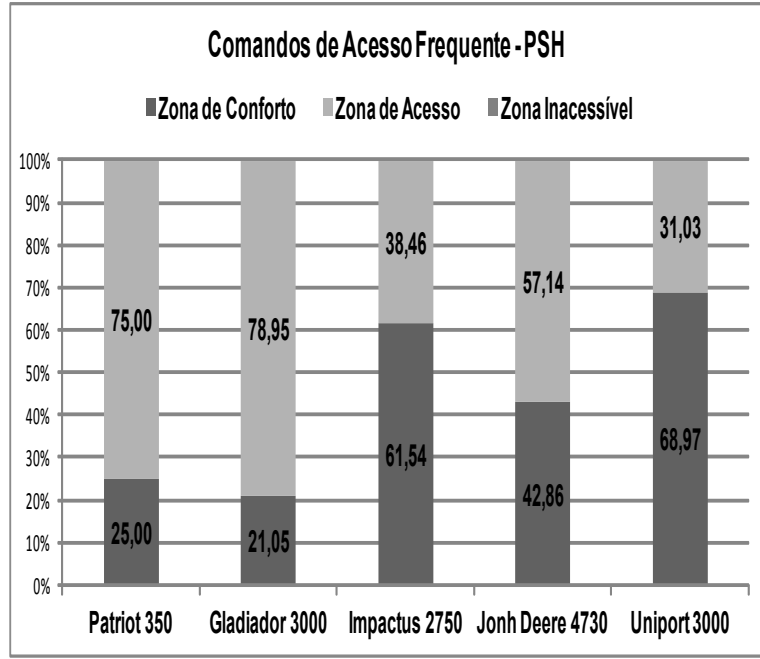

A

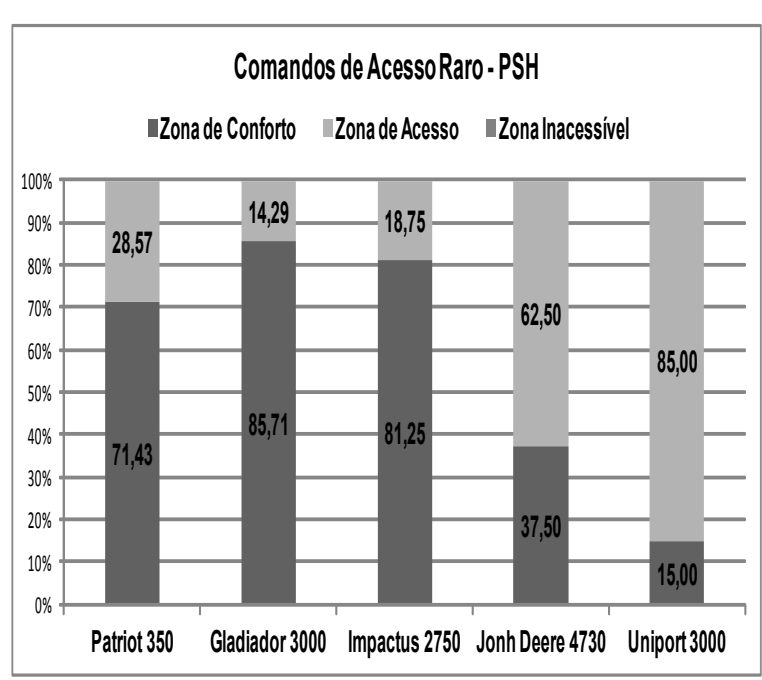

B

Figura 9. Distribuição dos comandos de acionamento frequente (A) e raro (B) no PSH para os cinco pulverizadores avaliados.

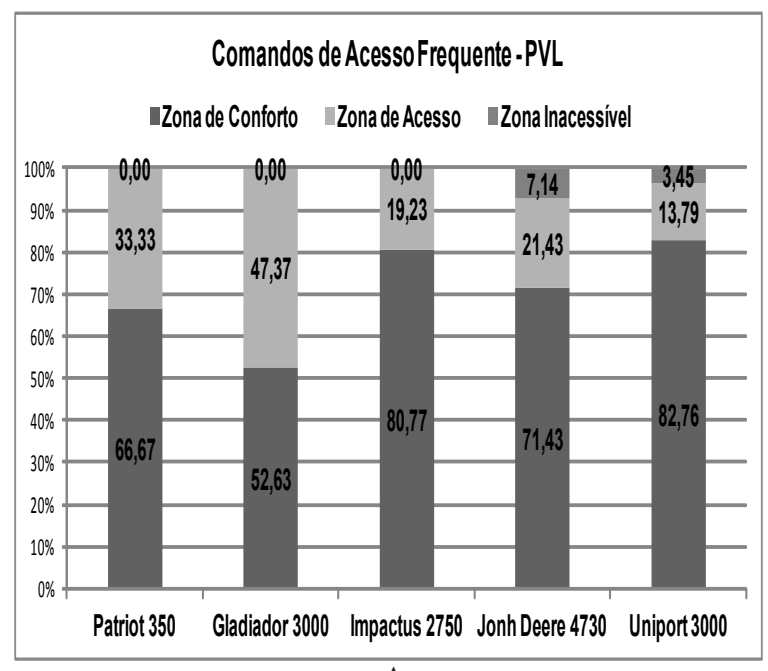

A

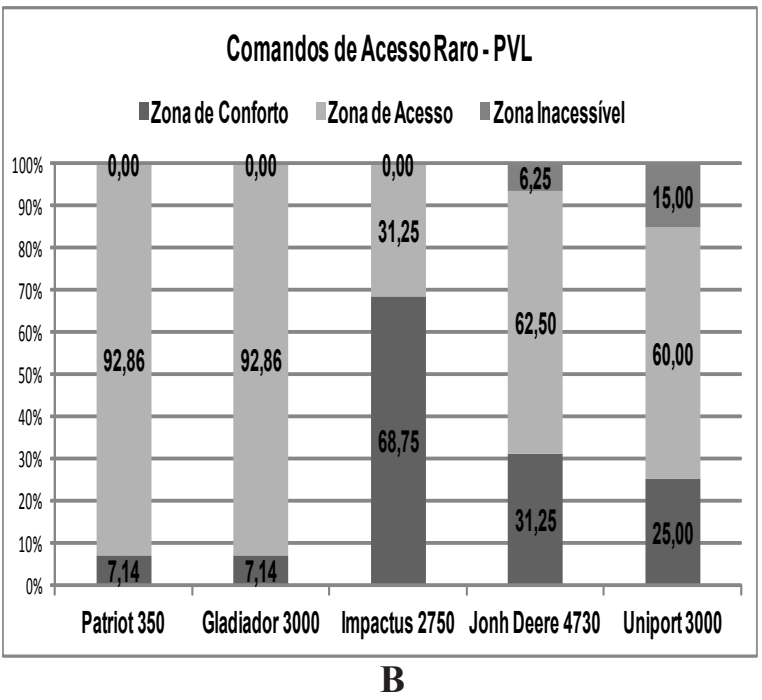

B

Figura 10. Distribuição dos comandos de acionamento frequente (A) e raro (B) no PVL para os cinco pulverizadores avaliados.

Na análise do PVL, os comandos frequentemente acionados estavam presentes em maior número na zona de conforto nos pulverizadores Uniport 3000 e Impactus 2750 , com $82,76 \%$ e $80,77 \%$, respectivamente. Jánazona deacesso, o pulverizador Gladiador 3000 obteve $47,37 \%$ dos comandos. Com relação aos comandos de acesso raro, ambos os pulverizadores, Patriot 350 e Gladiador 3000, apresentaram $92,86 \%$ dos comandos de operação na zona de acesso, mostrando conformidade com a norma ISO 15077. O pulverizador Impactus 2750 foi o que apresentou a maior percentagem de todos os comandos, tanto os de acesso frequente como os de acesso raro, nos dois perfis, superior horizontal e vertical longitudinal, na zona de conforto para o operador. No PVL, dois pulverizadores apresentam comandos de acesso frequente e raro na zona inacessível (Figura 10).

Segundo Minette et al. (2008), todas as máquinas apresentam alguma desconformidade em relação à localização de seus comandos de operação. Isso se explica devido ao fato de os padrões para dimensões do posto de trabalho serem estabelecidos de acordo com as normas europeias, para operadores com 
até $190 \mathrm{~cm}$ de altura, em assento fixo com altura de $45 \mathrm{~cm}$ e ajustável para $5 \mathrm{~cm}$, podendo diferir das medidas representativas de operadores de outros países (Siqueira, 1976). Mesmo em meio a desconformidades no posicionamento de alguns comandos de operação, tendo como base o perfil dos operadores brasileiros, os atuais projetos de máquinas agrícolas mostram a crescente elevação do padrão de qualidade no que tange à ergonomia e à segurança dos tratores agrícolas fabricados no país. Isso também se aplica aos pulverizadores Impactus 2750, Patriot 350 e Gladiador 3000, que não apresentaram comandos localizados na zona inacessível em nenhum dos dois perfis analisados. Fontana et al. (2004) observam que em máquinas mais modernas é comum que a maior parte dos comandos de operação se situem entre as zonas de conforto e de acesso, expressando a evolução dos projetos de máquinas agrícolas no que tange à ergonomia e à segurança. Isso se comprova nos pulverizadores Case Patriot 350 e John Deere 4730 , pois no comparativo entre os comandos de acionamento frequente, que estavam presentes em todos os pulverizadores, esses dois modelos apresentaram $100 \%$ dos comandos dentro da zona de conforto.

\section{CONCLUSÕES}

- Os pulverizadores apresentam na maioria dos casos comandos de acionamento frequente dentro da zona de conforto;

- Os pulverizadores que mais atenderam às exigências da norma ISO 15077, considerando os comandos de acesso frequente em comum nos cinco modelos, foram os modelos Case Patriot 350 e John Deere 4730;

- Considerando todos os comandos de acionamento frequente em relação ao perfil superior horizontal, os pulverizadores Uniport 3000 e Impactus 2750 se destacaram por apresentarem o maior número de comandos na zona de conforto; e

- No perfil vertical longitudinal, a análise mostrou a mesma tendência, apontando os modelos Uniport 3000 e Impactus 2750 com o maior número de comandos de acesso frequente dentro da zona de conforto.

\section{REFERÊNCIAS BIBLIOGRÁFICAS}

ASSOCIAÇÃO BRASILEIRA DE NORMAS TÉCNICAS. Tratores agrícolas - Acomodação do assento do operador - Dimensões: NBR ISO 4253. Rio de Janeiro, 1999.

\section{BERASATEGUI, M. R. El asiento en los tractores} agrícolas. Madrid: Laboreo, 1997.

FONTANA, G.; SILVA, R. P.; LOPES, A.; FURLANI, C. E. A. Avaliação de características ergonômicas no posto do operador em colhedoras combinadas. Engenharia Agrícola, v.24, n.3, p. 684-694, 2004.

International Organization for Standardization. Tractors and machinery for agriculture and forestry - operator controls - Actuating forces, their displacement and location: ISO 15077-1996. Genève, 1996.

. Earth - moving machinery - Seat Index Point: ISO 5353-1978. Genève, 1978.

Agricultural Tractors - Operator's seating accommodation - Dimensions: ISO 42531993. Genève, 1993.

MINETTE, L. J.; SOUZA, A. P. de; SILVA, E. P. da; MEDEIROS, N. M. Postos de trabalho e perfil de operadores de máquinas de colheita florestal. Revista Ceres, v.55, n.1, p.66-73, 2008.

ROZIN, D. Conformidade do posto de operação de tratores agrícolas nacionais com Normas de ergonomia e segurança. 204p. Dissertação de Mestrado. Universidade Federal de Santa Maria, Santa Maria, 2004.

SIQUEIRA, C.A.A. Um estudo antropométrico de trabalhadores brasileiros. Rio de Janeiro: COPPE/UFRJ, p.53, 1976. 